\title{
Modelo de aprendizaje desarrollador de la informática para el preuniversitario cubano.
}

(c) (1) (2) (2) Check for updates

Developing learning model of the information technology for the Cuban pre-university student.

Walfredo González Hernández. ${ }^{1}$ \& Roberto Jesús Bueno Hernández. ${ }^{2}$

Recibido: 10-08-2021 / Revisado: 20-08-2021 /Aceptado: 07-09-2021/ Publicado: 05-10-2021

\begin{abstract}
:
DOI: https://doi.org/10.33262/cienciadigital.v5i4.1866

Learning is one of the processes to them that more attention is lent today for the nations. In a first moment the search of models that concern the developing learning of the information technology from the method of documentary analysis is discussed, at a later time it discusses the considerations about the developing learning y the developing learning of information technology y the fashion model finally sets for itself as solution to the objective.
\end{abstract}

Keywords: learning, developer learning, information technology development learning, model.

Resumen: El aprendizaje es uno de los procesos a los que más atención se les presta hoy en día por las naciones. En un primer momento se aborda la búsqueda de modelos de aprendizaje desarrollador de la informática a partir del método de análisis documental, posteriormente se discuten las consideraciones acerca del aprendizaje desarrollador y el aprendizaje desarrollador de la informática y por último se propone el modelo como solución al objetivo trazado.

\footnotetext{
1 Doctor en Ciencias Pedagógicas. Profesor en la Universidad de Matanzas, Matanzas, Cuba. walfredo.glez@umcc.cu, https://orcid.org/0000-0003-4028-4266

2 II. Máster en Matemática Educativa. Profesor en la Universidad de Matanzas, Matanzas, Cuba. roberto.bueno@umcc.cu, https://orcid.org/0000-0002-5573-0049
} 
Palabras clave: aprendizaje, aprendizaje desarrollador, aprendizaje desarrollador de la informática, modelo.

\section{Introducción}

La concepción predominante acerca del aprendizaje desarrollador propuesta por Castellanos Simons, Reinoso Cápiro y García Sánchez (2004) parte del concepto de aprendizaje sustentado en dos de las vertientes fundamentales del enfoque histórico cultural creado por el trío de Yarkov integrado por Luria, Leontiev y Vygostki (Yasnitsky, 2012). Una de estas vertientes es reconocida como teoría de la actividad elaborada por A. N. Leontiev (Barahon, 2020) y la otra como Teoría de la Personalidad propuesta por L.I. Bozhovich (2009). La concepción de aprendizaje desarrollador posee tres dimensiones fundamentales: Activación - regulación, significatividad y motivación por aprender. Esta concepción del aprendizaje no tiene determinadas categorías del enfoque histórico cultural y que no son tenidos en cuenta como es la situación social del desarrollo L. I. Bozhovich (2009). Como se puede apreciar, desde esta autora, cada período de desarrollo conlleva análisis diferentes. Al mismo tiempo, el aprendizaje desarrollador explicado hasta el momento realiza un análisis de los estudiantes divididos en dimensiones e indicadores perdiendo de vista unidades integradoras de lo cognitivo y lo afectivo en la personalidad.

Otra concepción acerca del aprendizaje desarrollador lo plantea como un

... proceso complejo que implica al sistema de sentidos subjetivos asociados con la apropiación del contenido de enseñanza, sobre cómo se expresan sus configuraciones subjetivas en la rama del saber humano con sus formas de actividad fundamentales, en interacción no lineal con el resto de los sistemas que intervienen (González-Hernández, 2018, p. 22).

Esta concepción se basa en la tercera vertiente del enfoque histórico cultural desarrollado por el psicólogo cubano Fernando González Rey (Magalhães Goulart, Patiño Torres y Mitjáns Martínez 2020). Esta teoría se basa en 5 categorías fundamentales que explican al ser humano: sentido subjetivo, configuración subjetiva social e individual, subjetividad y sujeto. Desde estas categorías proveen una comprensión más holística e integradora de la personalidad que elimina las dicotomías entre lo interno-externo, individual-social, entre otras al concebir la personalidad como una configuración de configuraciones (Goulart, González Rey y Patiño Torres, 2019). Desde esta vertiente del enfoque histórico cultural González-Hernández (2018) considera que el aprendizaje desarrollador posee dos dimensiones: subjetivado y configuracional. Considerar este aprendizaje asumiendo estas dos dimensiones logra desterrar las divisiones entre los procesos afectivos y cognitivos, entre lo social y lo individual e integra en análisis configuracionales a la familia, la comunidad y la escuela para el análisis de la actuación del estudiante. 
La informática es una de las ciencias que mayor auge ha tenido en su desarrollo durante los últimos tiempos y su desarrollo se expresa a través de tecnologías. Esta ciencia ha logrado penetrar en las más disímiles ramas humanas, incluso creando nuevas ramas del saber humano como la bioinformática. Ello ha llevado a que su aprendizaje esté entre las prioridades en muchos países como una vía para lograr su desarrollo (Kombe, 2020). De ahí que se asuma como aprendizaje desarrollador de la informática la definición dada por González-Hernández (2019, p. 113) “... un proceso configuracional que implica al sistema de sentidos subjetivos asociados con la apropiación del contenido a aprender, sobre cómo se expresan sus configuraciones subjetivas en la informática en el proyecto como como configuración subjetiva social". La búsqueda vías para el aprendizaje desarrollador de esta ciencia es una prioridad en las estrategias de los países y Cuba no está exenta de esto. Las entrevistas realizadas a directivos de la enseñanza de esta ciencia arrojan las siguientes fortalezas y debilidades.

- Fortalezas:

- Existencia de una política a nivel de país encaminada a fortalecer la enseñanza de la informática en todos los niveles educativos.

- Proceso de perfeccionamiento escalonado de los planes de estudios en todos los niveles de enseñanza.

- Distribución de programas y libros de textos para la enseñanza de esta asignatura en todos los centros educativos del país.

- Debilidades

- Pocas investigaciones a nivel de país que guíen el proceder metodológico y tracen estrategias de para la enseñanza de esta ciencia.

- Pobres resultados de los estudiantes en concursos de informática a nivel nacional.

- Insuficiente aprendizaje de los contenidos de informática en los egresados del preuniversitario.

- Predomina un aprendizaje memorístico que no permite a los estudiantes un aprendizaje desarrollador de los contenidos informáticos del subsistema.

El preuniversitario, como parte del subsistema de educación cubano, adolece de estas insuficiencias. Este nivel se divide en tres años denominados décimo, onceno y duodécimo grado y su función fundamental es preparar a los estudiantes como futuros estudiantes universitarios. Aunque existe una variada literatura acerca de la enseñanza de estas disciplinas informáticas (Ahmad Basuhail, 2020; Luik y Lepp, 2021), lo disperso de esta literatura y la heterogeneidad de sus resultados hace que resulte es necesario un modelo que guíe las estrategias y principios de su enseñanza. Es por ello objetivo de este artículo elaborar un modelo de aprendizaje desarrollador de la informática para el preuniversitario cubano. 


\section{Método}

El análisis documental es un método muy utilizado en las investigaciones actuales (Zaragoza Vega y Gutiérrez Pérez, 2019) cuando se trata del análisis de documentos para establecer inferencias a partir de su contenido. En el caso de este artículo se aborda el análisis de la estructura de los modelos que permitan caracterizar el aprendizaje desarrollador de la informática en el preuniversitario cubano. Para la ejecución de este método los autores siendo el más completo encontrado en la literatura el utilizado por Zaragoza Vega y Gutiérrez Pérez (2019) compuesto por las siguientes fases: búsqueda de información, recuperación de los documentos, organización de la información, análisis crítico y detectar vacíos. Sin embargo, este tratamiento no tendría sentido sin una solución a la problemática que da origen a este proceso; por tanto, es importante añadir una fase denominada propuesta de solución. Como indica su nombre, esta última etapa propuesta debe describir la solución propuesta. También es necesario validar la propuesta de solución que se describe de tal manera que sea posible su aplicación. Cada una de las fases se han ajustado al modelo de artículo propuesto por la revista, las tres primeras fases del estudio corresponden al método, la cuarta y quinta parte corresponden a la propuesta de resultados y la propuesta de solución correspondería a la discusión. La correspondencia propuesta entre las fases del análisis documental y el formato IMRD pudiera ser tomado como pauta a seguir en otros artículos.

En la primera etapa se realizó una búsqueda de información en diferentes buscadores: sciencedirect.com y Scielo.com. Para ello se utilizaron las palabras claves: modelo, aprendizaje, desarrollador, informática en el título de los documentos que contienen estas bases de datos.

- https://www.sciencedirect.com/search?tak=developmental\%20learning\%20mod el\%20informatics

- (https://search.scielo.org/?q=***ylang=ptycount=15yfrom=0youtput=siteysort=y format $=$ summary $\mathrm{fb}=\mathrm{ypage}=1 \mathrm{yq}=*$ ylang $=$ ptypage $=1$

Segunda Etapa: Las palabras claves declaradas no dieron como resultado ningún ítem lo que permite afirmar que no se detectan investigaciones de este tipo. Para continuar la búsqueda de referentes acerca de los modelos se fueron eliminando palabras claves, la primera de ellas fue informática, posteriormente desarrollador. Con este proceso se obtuvieron más de 200 ítems de los cuales fueron seleccionados 68 por corresponder a modelos de aprendizaje o relacionados con el aprendizaje.

\section{Tercera Etapa}

Los documentos descargados fueron recopilados en una carpeta e importados a una biblioteca digital personalizada en el software EndNote X9 para lograr una mejor visualización, organización y selección acorde a las necesidades de este artículo. Las restantes etapas serán descritas en los siguientes apartados del artículo. 


\section{Resultados}

Los investigadores utilizan los modelos para obtener inferencias a partir de las abstracciones que hacen de la realidad y ellos permiten la generalización del conocimiento. Para algunos autores (Molina Hernández, González Hernández y Cruz Lemus, 2021) los modelos pueden basarse en alguna metodología particular como el aprendizaje basado en proyectos o patrones de aprendizaje. Sin embargo, este razonamiento pudiera limitar los niveles de generalización para aplicar el proyecto pues se limita a un aspecto específico. Para Davis (2020, p. 58-59) un modelo “... es la representación que se hace del objeto de investigación para resolver el problema objeto de análisis, como instrumento para optimizar el proceso, que tiene en cuenta el lugar del profesor, el de los estudiantes y el del grupo"

El análisis del artículo propuesto por Davis (2020) los modelos presentan una estructura compuesta por bases teóricas, procesos de aprendizaje, aspectos pedagógicos y teorías de aprendizaje que la sustentan. En este autor no se especifican qué entender por bases teóricas y los aspectos pedagógicos pues de esa manera parecen muy ambiguos. De otra manera Ndala (2020) propone un modelo para la educación superior en el que se proponen fundamentos filosóficos, psicológicos y didácticos mientras que para los autores Castro Sánchez et al. (2019, p. 9) los modelos didácticos “...presenta una estructura que incluye fin, objetivos, principios, caracterización del objeto, y formas de evaluación del modelo y formas de implementación del modelo". En este caso es importante tener en cuenta todos estos componentes del modelo didáctico en un modelo de aprendizaje.

Sin embargo, es necesario incluir otros elementos que se pueden observar en Flores (2019). Para este autor, un modelo debe estar compuesto por 11 elementos que permita su conocimiento desde posiciones teóricas así como su aplicación en la práctica:

1) un contexto socio-histórico de surgimiento del modelo; 2) una definición conceptual, 3) su fundamento filosófico epistemológico; 4) el fundamento psicológico, 5) los fines de la educación, 6) contenidos del currículo, 7) noción de aprendizaje, 8) rol del estudiante, 9) rol del maestro, 10) estrategias de enseñanza y 11) dispositivos de evaluación" (p. 149).

Es por ello que se asume en este artículo la última estructura añadiendo una última etapa de forma de implementación.

\section{Discusión}

Es necesario develar las interrelaciones entre los componentes del modelo, así como las particularidades de cada uno de ellos. Una representación esquemática se encuentra en la Figura 1 que se presenta a continuación: 


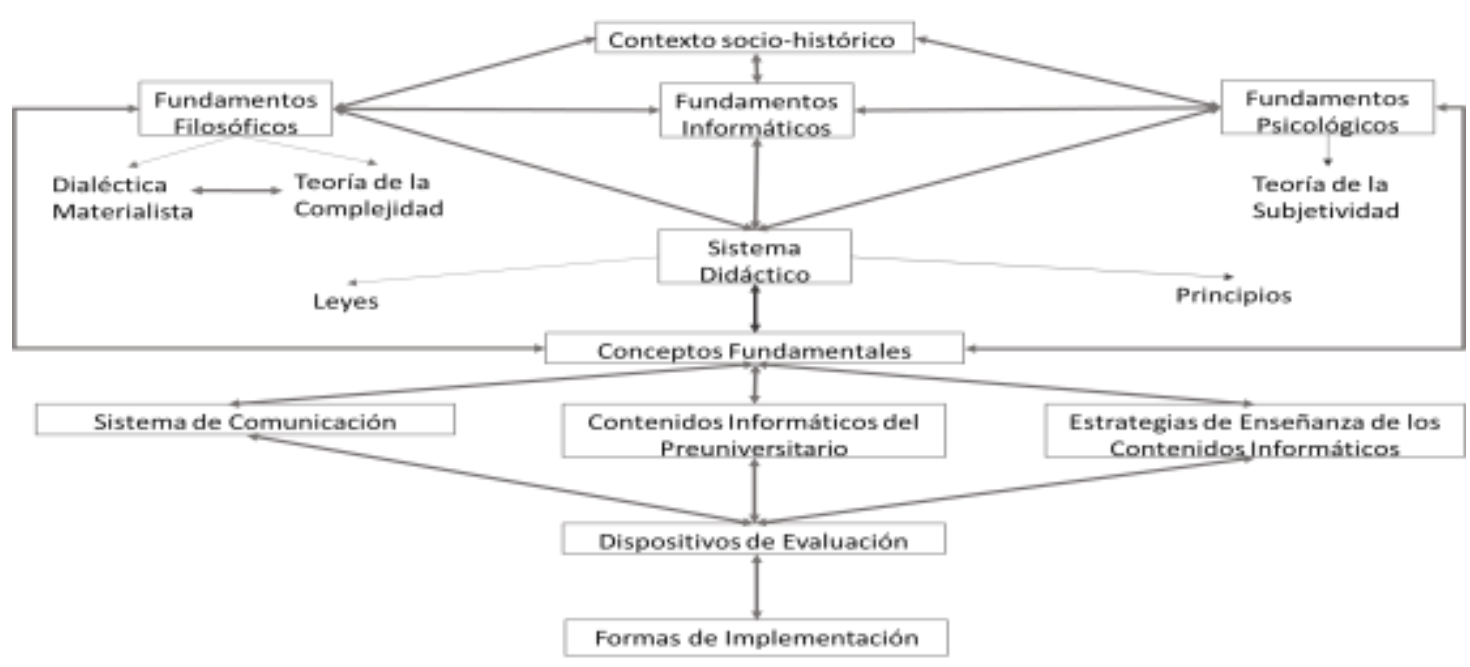

Figura 1: Estructura del Modelo de Aprendizaje Desarrollador de la Informática en el Preuniversitario. Fuente: Elaboración del Autor

Contexto socio-histórico de surgimiento del modelo

El contexto actual se caracteriza por un nivel alto de penetración de la informática en la mayoría de las actividades humanas. Ellas contribuyen a agilizar los procesos humanos y a incrementar la calidad de los servicios que se prestan. En muchas ocasiones sustituyen al ser humano en actividades para las cuales su constitución física no está preparada. Debido a estas razones, la informatización de las sociedades es un tema de seguridad nacional para muchos países y la alfabetización digital de los ciudadanos es un tema recurrente (González Hernández, 2020). La enseñanza de la informática debe tener en cuenta que los contenidos que se enseñan tienen un nivel de obsolescencia muy alto con respecto a otras asignaturas. El desarrollo de nuevas versiones, la feroz competencia en el mercado de sistemas y tecnologías, así como la existencia de dos filosofías contrarias en el uso de las tecnologías: libre y propietaria hacen que la enseñanza de la informática deba centrarse en los conceptos de una familia de sistemas y no aquellos conceptos particulares.

Todo modelo posee fundamentos filosóficos que pueden ser declarados o no explícitamente. En el caso de este modelo se fundamenta desde la integración de la dialéctica materialista por un lado que aporta sus leyes y principios mientras que por el otro se encuentra la teoría de la complejidad que aporta los elementos necesarios para realizar los análisis multifactoriales de los fenómenos complejos. La educación es analizada por varios autores como un proceso de alto grado de complejidad (Pennings, 2017) en el que las acciones educativas que hoy pudieran tener una alta eficacia, mañana pueden ser contraproducentes. Este fenómeno hace pensar que la los procesos educacionales, al decir de (González-Hernández, 2016a), son configuracionales porque cada uno de ellos modifica su estructura en dependencia de los flujos informacionales que establece con otros sistemas complejos en diferentes entornos. Las relaciones que establecen los profesores con los diferentes grupos de estudiantes, la concreción del acto didáctico, la elaboración de un currículo son ejemplos de procesos complejos en la 
educación así como la Teoría de la Subjetividad que constituye el fundamento psicológico de este modelo (Magalhães Goulart, 2019).

González Rey (2018) propone la teoría de la subjetividad y se constituye en una de las tres vertientes fundamentales del desarrollo en el enfoque histórico cultural iniciado por Lev Semionovich Vigotsky. Para González-Rey y Magalhães Goulart (2019) los sentidos subjetivos emergen durante la actuación del individuo como unidades simbólicos emocionales y se integran en configuraciones subjetivas. Estas configuraciones subjetivas expresan la historicidad en el desarrollo humano al mismo tiempo que logra la unidad de lo consciente y lo inconsciente. Llevada a los procesos de aprendizaje, estas categorías permiten analizar al estudiante como un sujeto activo, emocional y consciente. De ahí que se pueda explicar uno de los procesos más importantes de esta etapa, los intereses profesionales, como una configuración subjetiva. Los intereses profesionales comienzan desde edades tempranas cuando le niño comienza a preguntarse qué quiero hacer y la emergencia de sentidos subjetivos favorables a determinadas actividades va trazando el curso de estos intereses hasta que se asocian a alguna profesión o a un conjunto de ellas.

Los fundamentos informáticos del modelo se encuentran en los contenidos del plan de estudios para este subsistema educativo: hipermedias, bases de datos y programación fundamentalmente. Cada uno de estos contenidos contribuye a un proceso de informatización de la comunidad al poder establecer bases de datos sencillas para el manejo de las informaciones de los cuentapropistas que deben obtener para su negocio, para el manejo de las informaciones de la propia escuela como puede ser informatizar la secretaría docente, entre otras. De la misma manera pueden surgir proyectos para las asignaturas y lograr interdisciplinariedad con las asignaturas en las que el estudiante esté interesado en continuar sus estudios universitarios.

Las leyes del proceso asumidas son:

La relación configuracional entre la sociedad y la educación (González-Hernández, 2016a): Esta ley plantea que en los procesos educativos existe la tensión entre las necesidades de los estudiantes, las expectativas de los profesores y los objetivos planteados en su plan de estudios. En el caso de la enseñanza de la informática, los objetivos del plan de estudios en el preuniversitario pueden ser analizados por los estudiantes como poco interesantes o difíciles de cumplir por el nivel de abstracción que poseen. Las representaciones sociales que existan en cada grado acerca de la asignatura o los modos de actuación del profesor se transmiten de año en año y pueden añadir tensión a las ya existentes. La solución a estas tensiones conduce a un aprendizaje desarrollador, cuando no hay solución se produce un aprendizaje predominantemente memorístico.

Sistema complejo compuesto por varios subsistemas con interacciones configuracionales (González-Hernández, 2016a): El acto didáctico es el espacio donde se configuran todos los objetivos, contenidos, métodos y medios del proceso didáctico a partir de las interacciones que ocurren entre los componentes personales del proceso. El acto didáctico debe ser planificado, ejecutado y controlado por el profesor a partir de un proceso complejo de transposiciones didácticas que determinan los contenidos a enseñar y se van 
estructurando desde el saber informático hasta el saber que aprenden los estudiantes por un proceso de transposición didáctica. El sistema de interacciones que se dan en estos subsistemas es no lineal y en ellas existen tensiones entre sus participantes lo que impacta en los procesos de aprendizaje.

Los principios se sintetizan en los siguientes (González-Hernández, 2021):

El principio del carácter subjetivo del aprendizaje: este principio propone al aprendizaje como un proceso en el cual emergen sentidos subjetivos que se integran en configuraciones subjetivas sociales e individuales en todos los componentes personales del proceso. Ello implica que todos los espacios y unidades didácticas deben estar diseñados para que sus participantes generen producciones subjetivas sociales e individuales. Para este propósito la familia y la comunidad deben estar presente en todos los análisis pues tienen un peso importante en la formación.

El principio del carácter configuracional y complejo de educación: Los procesos educativos son esencialmente procesos complejos en todos los subsistemas y en este es de mayor complejidad pues cierra el ciclo de formación general y prepara a los estudiantes para el ingreso a la universidad. Cada uno de estos procesos que intervienen se integran de manera única en el que intervienen muchos factores que no siempre tienen la misma influencia. Uno de los factores con mayor nivel de tensión para este subsistema en Cuba lo constituyen las pruebas de ingreso a la universidad y la participación en concursos por las connotaciones que tienen.

Conceptos fundamentales del modelo:

El concepto de espacio de aprendizaje: este concepto permite integrar todos aquellos espacios donde el estudiante aprende más allá del aula como espacio de aprendizaje teóricamente privilegiado. La familia, la comunidad, las amistades, o sea, todos los espacios físicos o virtuales donde los estudiantes se implican como sujetos de su aprendizaje deben ser considerados. En estos espacios los estudiantes se reafirman como sujetos de su aprendizaje y los objetivos institucionales, como expresión de los resultados deseados del proceso de formación, dejan de ser cumplidos cuando la escuela deja de ser el espacio de aprendizaje que conduzca el desarrollo de los estudiantes. En este sentido, las pruebas de ingreso son uno de factores que intervienen con mayor fuerza en la búsqueda de nuevos espacios donde prepararse para lograr el ingreso a la universidad en una carrera que se corresponda con sus intenciones

El concepto de problema: El problema es de las categorías didácticas más estudiadas en la actualidad y es la base de muchos modelos de resolución que comenzaron con el artículo How to solve it (Polya, 2004). El aprendizaje basado en problemas (Pérez Pérez, 2021), la enseñanza problémica (Espinoza Freire, 2018), entre otros modelos educativos, son ejemplos de ello. Sin embargo, como bien se plantea en una amplia literatura al respecto en los últimos años (Bueno Hernández, González Hernández y Naveira Carreño, 2020; Martínez-Padrón, 2021), los modelos anteriores no abordan con suficiente profundidad los procesos afectivos que tienen lugar en la resolución de problemas. Una 
solución a esta problemática la plantean los autores Bueno Hernández et al. (2020) cuando definen que un problema es una “... producción subjetiva que sucede al interactuar con una situación de la cual emergen sentidos subjetivos que provocan tensiones con las configuraciones subjetivas constituidas en el individuo" (p. 116). Esta definición tiene en cuenta no sólo la situación que se le presenta en el momento sino todo el contexto social e histórico que rodea a la situación planteada a los estudiantes. Las asignaturas relacionadas con programación y bases de datos poseen una historia de complejas y difíciles que son transmitidas de un grado a otro pudiendo influir en la percepción que tienen los estudiantes acerca de la complejidad que pueden realmente tener.

Concepto de proyecto: Los proyectos informáticos han sido uno de los factores fundamentales en el desarrollo histórico de la informática (Anisimova, Ganeeva y Sharafeeva, 2021; Dias Couto, 2020) pues en él se integran las acciones para solucionar los procesos de informatización a organizaciones. Ello lleva a que existan disímiles definiciones (Anisimova et al., 2021; Pressman y Lowe, 2013), sin embargo, como bien reconoce en estos autores, las definiciones están asociadas a las acciones y elementos que deben ejecutarse para obtener un producto con calidad. Para esta investigación, los proyectos no son sólo acciones sino son espacios de aprendizaje que se constituyen para ejecutar acciones de informatización con determinadas tecnologías. Desde esta definición se potencia más los procesos de producción que de ejecución que llevan a los estudiantes a convertirse en sujetos de su aprendizaje.

\section{Los Contenidos Informáticos del Currículo}

Los contenidos de informática están estructurados por cada grado que compone el preuniversitario cubano según está estipulado en los programas de las asignaturas. Cada uno de los programas de las asignaturas poseen una estructura compuesta por:

- Caracterización de la asignatura en el grado: Este acápite plantea el contexto en que se debe desarrollar la asignatura, la caracterización del estudiante, así como el rol que plantea la asignatura en el sistema de asignaturas del año.

- Objetivos generales de la asignatura informática en el nivel medio superior: este acápite plantea los objetivos de las asignaturas de informática en este subsistema para que el profesor pueda comprender, de manera general, las relaciones que establece el contenido de su asignatura con el resto.

- Objetivos generales de la asignatura en el grado: Comprende los objetivos a lograr durante la enseñanza de los contenidos correspondientes al año.

- Plan temático: Se colocan los conocimientos que el estudiante debe aprender, así como el tiempo que el profesor puede dedicar a cada uno de ellos.

- Orientaciones o sugerencias generales por unidades: En estas orientaciones se ofrecen las pautas generales que consideran los autores deben ser tenidas en cuenta para la enseñanza. 
- Concepción de la evaluación de los estudiantes. Este acápite se dedica a explicar las evaluaciones a realizar, la concepción de cada una de ellas, así como lineamientos generales para su frecuencia y elaboración.

- Bibliografía para el docente: La bibliografía para el docente aporta un conjunto de materiales que permiten al docente profundizar en el contenido que va a enseñar.

Para Décimo Grado (Pérez González y Labañino Rizzo, 2016) se proponen dos temas que abordan los recursos mediáticos estáticos y los recursos mediáticos continuos. De manera general los estudiantes deben culminar el primer tema aplicando las buenas prácticas del diseño a imágenes a través del uso de una herramienta de tratamiento de imágenes. En el segundo tema deben aplicar las buenas prácticas del diseño a videos y animaciones a través del uso de una herramienta de tratamiento de video. Las herramientas a utilizar se determinan por el sistema educativo teniendo en cuenta la infraestructura escolar.

En el caso de Onceno Grado se plantean tres temáticas, un primer tema sobre las redes sociales, un segundo tema relacionado con las bases de datos y un tercer tema con los principios de la programación. En el primer tema se desglosan un sistema de objetivos que están orientados a la creación de una cultura informacional para el tratamiento de las informaciones que reciben desde las redes sociales. En el segundo tema deben aplicar los conceptos básicos del diseño de las bases de datos a situaciones prácticas a través del uso de un sistema gestor de bases de datos y en el tercer tema deben implementar soluciones a situaciones en las que apliquen las técnicas de programación utilizando un lenguaje. Al igual que en la anterior, el sistema educativo determina las herramientas para el cumplimiento de los objetivos trazados en cada tema.

En el caso de Duodécimo Grado se dedican 10 horas a los gestores y 40 horas a continuar el estudio de la programación. En el primer tema los estudiantes deben organizar una biblioteca digital personalizada utilizando un gestor bibliográfico que les permita la organización de la información en una temática dada. Durante el segundo tema profundizan en el estudio de las técnicas de programación al aplicar el modelo orientado a objetos en la solución de situaciones utilizando un lenguaje orientado a objetos.

Estos contenidos cuentan con un amplio respaldo bibliográfico en internet desde la que se proveen cursos online y variados recursos digitales que les permiten a los estudiantes profundizar en ellos. Ello posibilita que los estudiantes puedan acceder a ellos desde instituciones no escolares en los cuales pueden profundizar.

Sistema de comunicación: los sistemas de comunicación basados en el diálogo han demostrado su eficiencia en los procesos de aprendizaje (González-Rey, 2019). A través de su utilización como vía fundamental para mitigar las tensiones y la aceptación de los otros como son. La construcción de un diálogo franco que no penalice el error y valore las aportaciones de cada uno, contribuye a la emergencia de sentidos subjetivos favorables al aprendizaje. El apoyo del profesor para la corrección de los errores cometidos durante los procesos de algoritmización o diseño de bases de datos es vital. Para ello, un primer paso interesante estaría en detectar las concepciones que poseen los estudiantes acerca de 
su asignatura y actuación como profesor en relación con la complejidad de los conocimientos. Ello implica una reestructuración de los programas de resolución de problemas en general y, en particular, para la enseñanza de la informática. Para crear un clima favorable, el profesor debe crear condiciones de seguridad y apoyo que necesitan los estudiantes sin descuidar el principio del carácter científico de la enseñanza. Ello implica que el estudiante debe ir ganando confianza en su interacción con los conocimientos de la asignatura en la medida que se refuerzan sus éxitos, se minimizan los fracasos, se deja de ponderar el cuánto falta por llegar a la meta y se enfatiza en los logros que ha tenido. De esta manera se va construyendo una configuración subjetiva del aprender donde todos son importantes porque aportan soluciones informáticas desde los conocimientos que poseen a situaciones. Ello propicia que el aula se vaya convirtiendo en un lugar (Esteban-Guitart y Llopart, 2016) donde no sólo se aprenden conocimientos de programación, bases de datos o redes informáticas sino que se van construyendo normas, costumbres y prácticas. Esta construcción subjetiva de todos va adquiriendo un carácter único e irrepetible que no está exento de retrocesos ni tensiones en los que el diálogo juega un papel fundamental para su mitigación.

Estrategias de Enseñanza de los Contenidos Informáticos:

El preuniversitario, como subsistema de educación, tiene la misión de preparar a los estudiantes para su futuro ingreso a la universidad. Por tanto, la enseñanza de la informática debe prepararlo, desde los sistemas que utiliza en este nivel educativo, para enfrentar sistemas informáticos con versiones superiores debido a la rápida obsolescencia de los software. Una solución a esta disyuntiva educativa la provee la enseñanza basada en núcleos temáticos conceptuales (González-Hernández, 2016b) pues en su enseñanza se expresan los conceptos que definen la esencia de una familia de sistemas como son entidad, relación, variable, ciclo, objeto y clase. La agrupación de estos conceptos con sus procedimientos da origen a las líneas directrices para la enseñanza de la informática. En las orientaciones metodológicas de los programas de estudios se apunta a la enseñanza problémica como una vía propuesta para el aprendizaje desarrollador sin embargo, no se aclara ni en ellas ni en la bibliografía consultada (Espinoza Freire, 2018), cómo se logra que la situación problémica que presente el profesor estimule el estado deseado en los estudiantes. Otra alternativa propuesta en la literatura (Splichal, Oshima y Oshima, 2018) es el aprendizaje basado en proyectos y se reconoce por estos autores que promueve la independencia, la creatividad y el aprendizaje colaborativo. El aprendizaje basado en proyectos garantiza la individualización del aprendizaje pues cada estudiante posee un proyecto que debe concluir y en el que se expresan los conocimientos que debe aprender.

Teniendo en cuenta que el proyecto puede ser abarcador hasta completar todo el subsistema y puede integrarse con el enfoque de sistema que propone la integración de los núcleos conceptuales en líneas directrices (González-Hernández, Estrada-Sentí y Martínez-Llantada, 2006). La integración de los enfoques de enseñanza hace que emerjan sentidos subjetivos favorables hacia la programación pues les permite a los estudiantes ejecutar pequeños fragmentos de código en la computadora y observar los resultados de su trabajo. Sin embargo, es importante introducir el trabajo con algoritmos en forma de 
diagramas de bloques o lenguaje natural que les permita verificar si es la solución más eficiente. La necesidad de la eficiencia algorítmica se comprende a partir de la utilización de diversos códigos que pueden ralentizar el equipamiento disponible por ellos. Al mismo tiempo, permite hacerle pequeñas pruebas al pseudocódigo que permitan verificar si realmente funciona. Estos algoritmos pueden ser escritos en diversas herramientas que permiten generar códigos en diferentes lenguajes de programación. Estos códigos generados pueden ser comparados para que los estudiantes observen las implementaciones de los conceptos en diferentes lenguajes de programación y establezcan diferencias. A partir de este tratamiento, comienzan a integrarse sentidos subjetivos en configuraciones asociadas al aprender a programar. En la medida que el estudiante va generando algoritmos, comprobando que son eficientes y se ejecutan en la computadora, él va asociando la programación con el éxito y los sentidos subjetivos que van emergiendo son cada vez más favorables a esta actividad.

Una cuestión necesaria de dilucidar es la selección del lenguaje de programación a utilizar para codificar los algoritmos que los estudiantes obtienen. Los lenguajes de programación poseen conceptos y sintaxis que pueden ser complejas para el programador que se inicia por lo que un criterio de selección es contar con abundante material de consulta. Otro de criterio interesante se encuentra en la selección de un IDE con interface amigable e intuitiva que potencien la emergencia de sentidos subjetivos favorables hacia él por la abundancia de recursos para el reconocimiento sintáctico de las expresiones del lenguaje que utilizan. El uso de evaluadores en línea (Luik y Lepp, 2021) pudiera ser una alternativa interesante en otros contextos, sin embargo las limitaciones de conectividad presentes en el preuniversitario cubano hacen desechar su uso extendido. El lenguaje de programación seleccionado debe cumplir con el requisito de ejecutarse sin dificultades en las computadoras existentes en las instalaciones escolares.

Cuando estos algoritmos están asociados a un proyecto real que emana de una situación de informatización existente, el estudiante siente la satisfacción de ser útil al cliente de su trabajo. El proyecto puede surgir de una necesidad de informatización de alguna asignatura relacionada con sus intereses vocacionales, logrando que el estudiante se implique aún más. Las entregas de pequeñas partes del proyecto al cliente van generando sentidos subjetivos favorables relacionados con el programar y el estudiante se va implicando emocionalmente con el proyecto a partir de la satisfacción del cliente con cada entrega. En la medida que el proyecto avance y el estudiante se va implicando, las búsquedas de respuestas a las dudas que él posee se amplían y va construyendo nodos de información e interacción con otras personas. En estos espacios el estudiante va tomando aquellos "lugares" en los cuales puede compartir información y vivencias relacionadas con la programación. Cuando estos "lugares" se convierten en fuente de aprendizaje entonces se está en presencia de un espacio de aprendizaje en el cual el estudiante se convierte en sujeto de su aprendizaje. En este espacio de aprendizaje el estudiante va transgrediendo aquellos conocimientos que recibe a partir de la personalización de la información que recibe de su espacio de aprendizaje y los aplica en su proyecto. El desarrollo del proyecto de programación se va desarrollando a partir del tránsito por los 
nodos problémicos que suponen los conceptos y procedimientos necesarios para su solución.

De esta manera el estudiante se va insertando en pequeños proyectos de desarrollo de sistemas que no tienen gran complejidad, pero que logran la emergencia de emociones positivas integradas a los procesos simbólicos propios de la programación. Estos proyectos van desmitificando las ideas acerca de la complejidad de la programación al mismo tiempo que el estudiante trabaja en colectivo buscando soluciones sociales a los proyectos. La integración en equipos es posible, siempre que estén bien delimitados los algoritmos que concluye cada uno de manera independiente. De la misma manera, los análisis hechos hasta ahora con la programación pueden ser usados en el caso de las bases de datos. La integración entre estos dos contenidos no es posible como política debido a que no es contenido de programación la conexión de los programas con las bases de datos.

Dispositivos de evaluación: en el caso del preuniversitario, como toda la enseñanza media, el sistema de evaluación está determinado por el programa de estudios y no puede ser alterado por el profesor. En este programa se establecen dos trabajos de control y un examen final. Sin embargo, el examen final se plantea que puede ser integrador por lo que puede ser la defensa pública del resultado del proyecto que han estado desarrollando los estudiantes. La entrega de la documentación para la defensa del proyecto debe contener el portafolio con las entregas que han realizado al cliente. Estos hechos avalan los criterios que tiene el cliente acerca del nivel de terminación del proyecto, así como el avance que han tenido los estudiantes. En la literatura acerca de los portafolios (OlivaresGarcía, García-Segura, Gutiérrez-Santiuste y Mérida-Serrano, 2020) se plantea que pueden servir de medio de reflexión al estudiante sobre lo que ha logrado y cómo lo ha hecho a partir del análisis de las evidencias recogidas acerca de su trabajo. La presentación de los resultados parciales en talleres en forma de ferias de ciencia e innovación tecnológicas permiten la utilización de la coevaluación al mismo tiempo que los estudiantes van evaluando cómo se evalúan ellos mismos: la metaevaluación. Los estudiantes potencian la presentación de las ferias como una vía para presentar resultados, recoger opiniones de otros estudiantes acerca de la marcha de su trabajos e ideas que le permitan hacer más eficientes las soluciones.

Los procesos de evaluación esbozados en el párrafo anterior privilegian de manera natural uno de los tipos de evaluación descritos en el programa de la asignatura: la evaluación frecuente. La evaluación deja de ser un proceso temido por los estudiantes y pasa a ser un proceso solicitado pues les incrementa la cantidad de ideas que ellos pueden implementar en su proyecto. De la misma manera los cortes parciales del proyecto pueden convertirse en trabajos de controles parciales deseados por los estudiantes. El profesor se convierte así en un gestor de muchos proyectos que va conduciendo ante la emergencia de una configuración subjetiva social e individual del aprender en un proyecto. El estudiante está orientado hacia la solución de su proyecto y el proceso evaluativo consiste en valorar el cumplimiento de las metas trazadas al inicio del curso. Ello significa que el estudiante va incorporando sentidos subjetivos asociados a la responsabilidad y la laboriosidad que debe caracterizar su labor como futuro estudiante universitario. 
Formas de implementación: las estrategias constituyen parte de las formas de implementación de los modelos y en este caso se asume una estrategia metodológica como primer momento en la preparación de los profesores para la conducción del proceso de aprendizaje. El objetivo de la estrategia metodológica es: Contribuir al desarrollo del aprendizaje desarrollador de los contenidos informáticos en el subsistema educativo cubano preuniversitario. Como parte de la estructura de esta estrategia se proponen las siguientes etapas:

- Primera etapa de diagnóstico y planificación: Esta etapa sirve como base para todas las restantes acciones pues en ella se prepara y ejecuta el diagnóstico que permitirá determinar las necesidades metodológicas que poseen los profesores. El resultado de este diagnóstico servirá de base para la planificación de las acciones de transformación en los involucrados y se encuentran cursos de superación a profesores, talleres metodológicos sobre los fundamentos del modelo, clases metodológicas demostrativas con grupos de estudiantes entre otras. Por la estrecha interrelación entre diagnóstico y planificación es que se unen las dos etapas.

- Segunda etapa de ejecución de acciones transformadoras con profesores: En esta segunda etapa se ejecutan las acciones planificadas en la etapa anterior para que se reviertan en una mejor conducción del aprendizaje desarrollador de la informática en este subsistema. En este sentido la preparación didáctica e informática del profesor es objeto de amplios análisis (Corrales, Herrera y Martín, 2016; González Velázquez y Pedraza Rodríguez, 2019). La preparación metodológica de los profesores debe estar contextualizada a su quehacer diario como profesor de informática en el preuniversitario. Por ello los cursos de superación sólo deben abordar los elementos esenciales y ser de corta duración, de manera que el profesor pueda dedicar tiempo al análisis práctico de los procesos bajo la guía del investigador. Ello lleva al profesor a la búsqueda de información y la ejecución de investigaciones que le permita dar soluciones a las problemáticas que encuentre en su práctica profesional.

- Tercera etapa para el control y evaluación de los resultados: esta etapa es transversal a toda la estrategia y se aplica durante el resto de las acciones. Los directivos deben ser informados de los resultados que se van obteniendo durante la etapa de ejecución de las acciones. Es válido introducir acciones correctivas en caso que algunas de las acciones planificadas no estén obteniendo los resultados deseados. Ello lleva a la retroalimentación continua de todos los actores, en especial los profesores.

Este modelo muestra una alternativa factible que abre espacios de inteligibilidad en la enseñanza preuniversitaria que logra la emergencia de una configuración subjetiva social favorable al aprendizaje de los contenidos informáticos del grado. También demuestra la existencia de relaciones no lineales que expresan realidades en toda su complejidad. El modelo coloca en el centro del proceso de aprendizaje al estudiante, portador y productor de subjetividades que se integra en espacios de aprendizaje donde se implique como 
sujeto. Esta implicación debe ser lograda por el maestro de informática a partir de su actuación como guía del proceso de construcción de una comunidad de aprendizaje

\section{Referencias}

Ahmad Basuhail, A. (2020). Application of Learning Objects for Computer Programming-Based Problem Solving. Canadian Journal of Learning $y$ Technology, 46(1), 1-15.

Anisimova, T., Ganeeva, A. y Sharafeeva, L. (2021). Development of Digital Skills y Engineering Thinking in Students as Part of the Digital Summer Project. International Journal of Engineering Pedagogy, 11(2), 69-81. doi:10.3991/ijep.v11i2.17215

Barahon, M. (2020). Developing y enacting professional pedagogical responsibility: a CHAT perspective. The European Journal of Applied Linguistics y TEFL, 9(2), 131-152.

Bozhovich, L. I. (2009). The Social Situation of Child Development. Journal of Russian y East European Psychology, 47(4), 59-86. doi:10.2753/rpo1061-0405470403

Bozhovich, L. I. (2009). The Struggle for Concrete Psychology y the Integrated Study of Personality. Journal of Russian y East European Psychology, 47(4), 28-58.

Bueno Hernández, R. J., González Hernández, W. y Naveira Carreño, W. J. (2020). Análisis crítico acerca de la resolución de problemas desde la teoría de la subjetividad. Alternativas cubanas de psicología, 8(23), 112-125.

Castellanos Simons, D., Reinoso Cápiro, C. y García Sánchez, C. (2004). Para Promover un Aprendizaje Desarrollador. Ciudad de la Habana-Cuba: Editorial Pueblo y Educación.

Castro Sánchez, F., Toro Toro, J., Carrera Calderón, F., Mantilla Cabrera, L. F., Carrillo Rodríguez, M. A. y Ricardo Velásquez, M. (2019). Modelo didáctico para contribuir al desarrollo de la autonomía en el aprendizaje del inglés en la formación inicial de profesores de la carrera de lenguas extranjeras. Revista Dilemas Contemporáneos: Educación, Política y Valores, VI(2), 26.

Corrales, L., Herrera, J. y Martín, D. (2016). La superación profesional de los docentes universitarios para el perfeccionamiento de la dirección del aprendizaje. Márgenes, 3(3), 327-346.

Davis, V. (2020). Modelos didácticos para el aprendizaje de las disoluciones en la gerencia del aula. Gerentia(1), 221-241.

Dias Couto, P. (2020). Scrum e Personal Software Process: um framework de gestão de projeto para desenvolvedores standalone. (Bacharel em Engenharia da Computação), Pontifícia Universidade Católica de Goiás, Goiânia-Brazil. 
Espinoza Freire, E. E. (2018). Presencia de los métodos problémicos en la educación básica. Mendive: Revista de Educación, 16(2), 262-277.

Esteban-Guitart, M. y Llopart, M. (2016). La inclusión educativa a través de la aproximación de los fondos de conocimiento e identidad. Revista nacional e internacional de Educación Inclusiva, 9(3), 145-157.

Flores, M. (2019). Cuatro formas de entender la educación. Educación y Humanismo, 21(36), 137-159. doi:10.17081/eduhum.21.36.3147

González-Hernández, W. (2016a). Las leyes de la didáctica y la realidad escolarizada. ¿Necesidad de cambio? Revista Latinoamericana de Estudios Educativos, $\operatorname{XLVI(3),85-110.~}$

González-Hernández, W. (2016b). Propuesta metodológica para el tratamiento de conceptos y definiciones informáticos. Revista Electrónica Formación y Calidad Educativa (REFCalE), 4(2), 45-62.

González-Hernández, W. (2018). Aproximación al aprendizaje desarrollador en la Educación Superior. Educação (FSMG), 43(1), 11-26. doi:10.5902/1984644429309

González-Hernández, W. (2019). Definición del aprendizaje desarrollador de la informática por el profesional informático. Revista Educación en Ingeniería, 14(27), 106-115. doi:10.26507/rei.v14n27.969

González-Hernández, W. (2021). Didactic principles: A proposal from the theory of subjectivity. Culture y psychology. doi:10.1177/1354067x20984355

González-Hernández, W., Estrada-Sentí, V. y Martínez-Llantada, M. (2006). El enfoque de sistema en la enseñanza de la informática para el desarrollo de la creatividad. Revista Enseñanza Universitaria(26), 7-21.

González-Rey, F. (2019). Methodological y Epistemological Demands in advancing the study of subjectivity. Culture y Psychology, O(0), 1-16.

González-Rey, F. y Magalhães Goulart, D. (2019). Teoria da Subjetividade e educação: entrevista com Fernando González Rey. Obutchénie: Revista de Didatica e Psicologia Pedagógica, 3(1), 13-33.

González Hernández, D. (2020). Alfabetización mediática y producción práctica en medios: la edición digital desde la perspectiva de la cognición distribuida. Anuario de Investigación CONEICC, I(XXVII), 163-174.

González Rey, F. L. (2018). Vygotsky’s “The Psychology of Art”: A foundational y still unexplored text. Estudos de Psicologia, 35(4), 339-350. doi:10.1590/198202752018000400002 
González Velázquez, V. E. y Pedraza Rodríguez, E. M. (2019). Becas internacionales de investigación en Cuba: espacios para la superación científica estudiantil. Educación Médica, in press. doi:10.1016/j.edumed.2019.09.003

Goulart, D. M., González Rey, F. y Patiño Torres, J. F. (2019). The study of the subjectivity of mental health professionals: an experience in Brasília. Athenea Digital. Revista de pensamiento e investigación social, 19(3), 1-21. doi: $10.5565 / \mathrm{rev} /$ athenea.2548

Kombe, C. (2020). A secure y interoperable blockchain-based information sharing system for healthcare providers in developing countries. (Degree of Doctor of Philosophy in Information y Communication Sciences y Engineering), The Nelson Mandela African Institution of Science y Technology Arusha-Tanzania.

Luik, P. y Lepp, M. (2021). Are Highly Motivated Learners More Likely to Complete a Computer Programming MOOC? . International Review of Research in Open y Distributed Learning, 22(1), 41-58.

Magalhães Goulart, D. (2019). Subjectivity y life: in memory of Fernando González Rey. Mind, Culture, and Activity, 1-6. doi:10.1080/10749039.2019.1619775

Magalhães Goulart, D., Patiño Torres, J. F. y Mitjáns Martínez , A. (2020). Vida y legado de Fernando González Rey: introducción a la revista en su homenaje. Alternativas cubanas en Psicología, 8(23), 7-13.

Martínez-Padrón, O. J. (2021). El afecto en la resolución de problemas. Revista Caribeña de Investigación Educativa, 5(1), 86-100. doi:10.32541/recie.2021.v5i1.pp86100

Molina Hernández, C. R., González Hernández, W. y Cruz Lemus, G. (2021). Habilidad modelar procesos dinámicos de control automático. Educación Química, 32(1), 100-111. doi:10.22201/fq.18708404e.2021.1.75429

Ndala, D. (2020). Modelo didáctico de sistematización del contenido compuestos de coordinación de la química inorgánica. Magazine de las Ciencias: Revista de Investigación e Innovación, 5(5), 56-69.

Olivares-García, M. Á., García-Segura, S., Gutiérrez-Santiuste, E. y Mérida-Serrano, R. (2020). El e-portafolio profesional: una herramienta facilitadora en la transición al empleo de estudiantes de grado en educación social en la Universidad de Córdoba. Revista Española de Orientación y Psicopedagogía, 31(3), 129-148.

Pennings, H. J. M. (2017). Using a complexity approach to study the interpersonal dynamics in teacher---student interactions: a case study of two teachers. Complicity: An International Journal of Complexity y Education, 14(2), 88-103.

Programa de estudio para 10mo grado, (2016). 
Pérez Pérez, O. d.-J. (2021). Aprendizaje Basado en Resolución de Problemas (ABRP) como mediación didáctica para el desarrollo del razonamiento clínico y humanístico del residente de pediatría en una universidad del caribe colombiano. RIAICES, 3(1), 47-54. doi:10.17811/ria.2.1.2013.47-54

Polya, G. (2004). How to Solve It A New Aspect of Mathematical Method. PrincetonUSA: Expanded Princeton Science Library Edition.

Pressman, R. y Lowe, D. (2013). Web Engineering: A Practitioner's Approach: McGrawHill Higher Education.

Splichal, J. M., Oshima, J. y Oshima, R. (2018). Regulation of collaboration in projectbased learning mediated by CSCL scripting reflection. Computers y Education, 125, 132-145. doi:10.1016/j.compedu.2018.06.003

Yasnitsky, A. (2012). Uma História da Psicologia da Gestalt Histórico-Cultural Vygotsky, Luria, Koffka, Lewin, e outros. Dubna Psychological Journal(1), 106109.

Zaragoza Vega, O. y Gutiérrez Pérez, M. P. (2019). Efecto de la certificación docente en el cambio de las prácticas pedagógicas. Un análisis documental. Diálogos sobre educación(19), 1-16. doi:10.32870/dse.v0i19.501

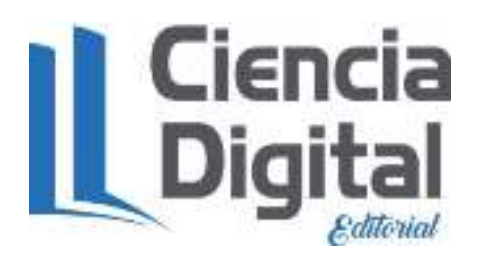




\section{PARA CITAR EL ARTÍCULO INDEXADO.}

González Hernández, W., \& Bueno Hernández, R. J. (2021). Modelo de aprendizaje desarrollador de la informática para el preuniversitario cubano. Ciencia Digital, 5(4), 27 45. https://doi.org/10.33262/cienciadigital.v5i4.1866

\section{Liencia}

El artículo que se publica es de exclusiva responsabilidad de los autores y no necesariamente reflejan el pensamiento de la Revista Ciencia Digital.

El artículo queda en propiedad de la revista y, por tanto, su publicación parcial y/o total en otro medio tiene que ser autorizado por el director de la Revista Ciencia Digital.
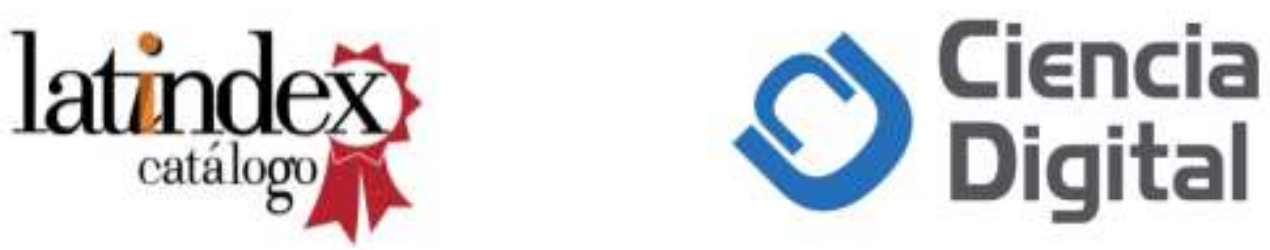